\title{
Early environmental enrichment enhances abnormal brain connectivity in a rabbit model of intrauterine growth restriction
}

Miriam Illaa $a^{a, b}$ Verónica Brito ${ }^{b-d}$, Laura Pla ${ }^{a, b}$, Elisenda Eixarcha, ${ }^{a, b, e}$, Ariadna Arbat-Plana $^{\mathrm{d}, \mathrm{f}}$, Dafnis Batalle ${ }^{\mathrm{g}}$, Emma Muñoz-Moreno ${ }^{\mathrm{a}, \mathrm{h}}$, Fatima Crispi ${ }^{\mathrm{a}, \mathrm{b}, \mathrm{e}}$, Esther Udina ${ }^{d, f}$, Francesc Figueras ${ }^{a, b, e}$, Sílvia Ginés ${ }^{b-d}$, Eduard Gratacós ${ }^{a, b, e}$

${ }^{a}$ Fetal i+D Fetal Medicine Research Center, BCNatal - Barcelona Center for Maternal-Fetal and Neonatal Medicine (Hospital Clínic and Hospital Sant Joan de Deu), Institut Clínic de Ginecologia, Obstetricia i Neonatologia, Universitat de Barcelona, Barcelona, Spain

b Institut d'Investigacions Biomèdiques August Pi i Sunyer (IDIBAPS), Barcelona, Spain

c Departament de Biomedicina, Facultat de Medicina, Institut de Neurociències, Universitat de Barcelona, Barcelona, Spain

d Centro de Investigación Biomédica en Red sobre Enfermedades Neurodegenerativas (CIBERNED), Bellaterra, Spain

e Centre for Biomedical Research on Rare Diseases (CIBER-ER), Barcelona, Spain

${ }^{f}$ Institute of Neurosciences, Department of Cell Biology, Physiology and Immunology, Universitat Autònoma de Barcelona, Bellaterra, Spain

${ }^{9}$ Centre for the Developing Brain, Division of Imaging Sciences \& Biomedical Engineering, King's College London, London, SE17EH, United Kingdom

h Experimental MRI 7T Unit, Institut d'Investigacions August $\mathrm{Pi}$ i Sunyer (IDIBAPS), Barcelona, Spain 


\section{* Corresponding Author:}

Elisenda Eixarch, MD PhD.

Fetal i+D Fetal Medicine Research Center, BCNatal - Barcelona Center for Maternal-Fetal and Neonatal Medicine (Hospital Clínic and Hospital Sant Joan de Déu), IDIBAPS, University of Barcelona, Barcelona, Spain.

Sabino de Arana 1, 08028 Barcelona, Spain.

Tel. +34 932275400 EXT. 7339 .

Email: eixarch@clinic.ub.es

*Short title: Environmental enrichment enhances brain connectivity in IUGR

\section{* Conflicts of Interest:}

The authors report no conflict of interest.

\section{* Source of Funding:}

The research leading to these results has received funding from "la Caixa" Foundation. Additionally, this study was supported by Instituto de Salud Carlos III (PI15/00130; PI12/00851; PI13/01018) integrated in the "Plan Nacional de I+D+I" and copaid by "ISCIII-Subdirección General de Evaluación" and "Fondo Europeo de Desarrollo Regional" (FEDER) "Una manera de hacer Europa"; AGAUR 2014 SGR grant n 928; Ministerio de Ciencia e Innovación (SAF2015 -67474-R;MINECO/FEDER to S.G); and Centro de Investigaciones Biomédicas en Red sobre Enfermedades Neurodegenerativas (CIBERNED). 


\section{ABSTRACT:}

Introduction: Structural correspondence of neurodevelopmental impairments related with Intrauterine growth restriction (IUGR) that persists later in life remains elusive. Moreover, early postnatal stimulation strategies have been proposed to mitigate these effects. Brain connectivity abnormalities at the long-term period in an IUGR rabbit model and the effects of early postnatal environmental enrichment have been explored.

Material and Methods: IUGR was surgically induced in one horn, whereas the contralateral produced the controls. Postnatally, a subgroup of IUGR animals was housed in an enriched environment. Functional assessment was performed at the neonatal and long-term periods. At the long-term period, structural brain connectivity was evaluated by means of diffusion brain resonance imaging and by histological assessment focused in the hippocampus.

Results: IUGR animals displayed poorer functional results and presented altered whole-brain networks and decreased median fractional anisotropy in the hippocampus. Reduced density of dendritic spines and perineuronal nets from hippocampal neurons were also observed. Of note, IUGR animals exposed to enriched environment presented an improvement in terms of both function and structure.

Discussion: IUGR is associated with altered brain connectivity at global and cellular level. A strategy based on early environmental enrichment has the potential to restore IUGR neurodevelopmental consequences. 


\section{Key words:}

Animal model, dendritic spine density, diffusion magnetic resonance, environmental enrichment, intrauterine growth restriction, object recognition task, open field behavioral test, perineuronal nets, Skinner test, therapy.

Abbreviations: DS: dendritic spine; EE: environmental enrichment; FA: fractional anisotropy; IUGR: intrauterine growth restriction; OFBT: open field behavioral test; ORT: object recognition task; PNNs: perineuronal nets. 


\section{INTRODUCTION}

Intrauterine growth restriction (IUGR) due to placenta insufficiency is a wellrecognized cause of perinatal morbi-mortality complications [1] along with neurobehavioral and cognitive impairments extending beyond childhood [2, 3] and early adulthood period [4-6]. These neurodevelopmental problems have not been only associated with severe cases, but also milder forms of IUGR are at risk for abnormal neurodevelopment [7]. While severe IUGR affects $3 \%$ of pregnancies, mild IUGR affects up to $7 \%$ of deliveries, that is about 600,000 cases in Europe [8], representing a huge public health issue. Actually, IUGR is considered, together with prematurity, as the cause of one-quarter of cases for special educational needs [9]. However, the structural ground of these functional impairments is not fully characterized.

The description of the brain changes underlying long-term neurodevelopmental impairments of IUGR is essential for the development of imaging biomarkers for early diagnosis, monitoring [10], and selection of specific therapeutic strategies. With the significant advance of magnetic resonance imaging (MRI) in the recent years, diffusion-weighted imaging (DWI) techniques have demonstrated altered brain network organization could play an important role in this disorder [11-14]. At the cellular level, standard histological assessment has not been able to show the structural brain changes underlying brain injury of IUGR that persist up to long-term period. More specific techniques focused on neuronal connectivity could better reflect the structural changes in IUGR, as changes in axonal and dendrite development have been suggested to be the histological basis of brain changes assessed by diffusion tensor imaging (DTI) studies [15,16]. 
IUGR might have long lasting consequences, and currently, breastfeeding has been demonstrated to be one of the more effective strategies to partially ameliorate the long-term neurodevelopmental sequelae of IUGR [17]. Recently, other therapies are now arising as promising strategies to overcome brain diseases. Environmental enrichment (EE) strategy has consistently been demonstrated to exert beneficial effects on stress conditions and cognitive impairments by improving complex cognitive functions [18] and animal's emotional and stress reactivity [19-21]. This functional improvement was accompanied by changes at the cellular level, in terms of increased dendritic arborization, the number of dendritic spines, synaptic density, and postsynaptic thickening, particularly in the hippocampus $[18,20,22]$. Moreover, it has been demonstrated that neural circuits display a heightened sensitivity from the external environment inputs in specific periods of early postnatal life [23]. However, the neurodevelopmental effect of early EE has never been evaluated in an animal model of IUGR.

Hence, in this study, we tested the hypotheses that 1) IUGR alters global and regional neuronal connectivity, and the changes persist up to the long-term period and, 2) a strategy based on EE applied during early postnatal period mitigates the neurodevelopmental impairments related to this condition. For this purpose, we evaluated neurobehaviour and structural changes in a rabbit model of placental insufficiency undergoing early EE [24]. Structural changes were assessed by advanced ex vivo DW-MRI and histological markers of neuronal connectivity, including dendritic spine (DS) density and perineuronal network (PNNs) in the hippocampus. 


\section{MATERIAL AND METHODS}

\section{Animals, IUGR induction, and Ethics Statement}

Animal experimentation was approved by the Animal Experimental Ethics Committee of the University of Barcelona (permit number: 553/13). IUGR was induced in 10 New Zealand pregnant rabbit dams following selective uteroplacental artery ligation procedure in one of the horns at 25 days of pregnancy as previously described [24], whereas the non-ligated contralateral horn produced the controls. The selection of the ligated horn was done at random. Five days later, a cesarean section was performed obtaining the animals. IUGR animals were obtained from the gestational sacs with arteries ligation, whereas the contralateral gestational sacs produced normally grown subjects (control). All surviving pups were weighed, identified by a subcutaneous microchip inserted in their back (Microchip MUSICC, Avid Microchip S.L., Barcelona, Spain) and were housed with a wet nurse rabbit with part of its offspring (maximum of 8 pups for each wet nurse), until the $30^{\text {th }}$ postnatal day when they were weaned.

\section{Environmental enrichment strategy}

After weaning ( $>30$ postnatal days), the animals were housed in groups of three with a reversed $12 / 12 \mathrm{~h}$ light cycle with free access to water and standard chow. The animals were housed in standard conditions, except for a subgroup of IUGR animals $(n=15)$ that were selected at random to be housed following an EE strategy (t-IUGR group). The designed EE protocol was based on previous knowledge of behavioral needs and data available from enrichment studies in rabbits [25]. The implemented strategy aimed to increase the animal sensory, 
physical, cognitive and social stimulation. For that purpose, the animals were housed in larger cages $(150 \times 70 \times 40 \mathrm{~cm})$ in comparison to the standard ones $(75 \times 70 \times 40 \mathrm{~cm})$. Inside both types of cages, an upper platform allowing the animal to look out was placed, as a basic environmental refinement. However, only inside the t-IUGR animals cage different inanimate objects (wooden bridge, colored balls, bricks) and different flavors of food were placed. Every three days the objects and the food were changed in order to induce novelty and cognitive stimulation. In addition, social stimulation was induced by placing the animals in

a big room for one hour twice per week, allowing them to freely explore the environment and to interact with a researcher (M.I., L.P.). This protocol was kept during 30 days up to the sacrifice of the animals. The study design is summarized in Figure 1.

\section{Functional tests protocol and sampling collection}

During the neonatal period (at +1 postnatal day), general motor skills, reflexes, and sensitivity were evaluated (controls: $n=24$; IUGR: $n=14$; t-IUGR: $n=15)$ as previously described by Derrick et al $[26,27]$.

At the long-term period ( +60 postnatal days), a set of neurodevelopmental tests was applied in both IUGR and t-IUGR animals (IUGR: $n=14$; $t$-IUGR: $n=15$ ). In the control group, due to the high number of animals eligible to be evaluated $(n=24)$, only a subsample of them was included in this analysis $(n=$ 13). In order to assess learning skills, Skinner test was applied following previously described methodology [28]. Learning was considered when the animal pressed the lever and went directly towards the food dispenser at least in three different times in the same session. For anxiety and memory 
evaluation, Open Field Behavioral Test (OFBT) and Object Recognition Task (ORT) were also applied [29]. ORT was attempted in all animals with a successful OFBT test, but only 11 controls, 14 IUGR, and 13 t-IUGR fulfilled the ORT's established criteria as previously suggested [30]. The SMART Software Tracking System (from Panlab Harvard Apparatus, UK) was used to record the variables from OFBT (time in seconds exploring the internal area) and ORT (time in seconds exploring familiar and novel objects). The cumulative time exploring both objects from the ORT was recorded and discrimination index (DI) was then calculated as follows:

$$
D I=\frac{\text { time exploring novel object }- \text { time exploring the familiar one }}{\text { time exploring novel object }+ \text { time exploring the familiar one }}
$$

A preserved memory was considered with $\mathrm{DI}>0$, whereas a $\mathrm{DI} \leq 0$ indicated problems in short-term memory. All functional tests were evaluated by two blinded observers (M.I, L.P).

\section{Sample collection}

After the neurobehavioral tests (at +70 postnatal days), rabbits were anesthetized with ketamine $35 \mathrm{mg} / \mathrm{kg}$ and xylazine $5 \mathrm{mg} / \mathrm{kg}$ given intramuscularly and were sacrificed with an endovenous overdose of sodium pentobarbital (200 mg/kg). Immediately after, 4 animals from each experimental group were randomly assigned to be included in the Dendritic spine (DS) evaluation and were processed according to this, whereas the rest of the animals followed a standard fixative protocol. In DS group, brains were fixed through cardiac perfusion with phosphate-buffered saline (PBS) followed by $2 \%$ paraformaldehyde (PFA), whereas the rest of the animals were fixed by $10 \%$ buffered $10 \%$ formalin. Finally, the cranial bone was removed and brains were 
also fixed by 10 minutes immersion in 2\% PFA in the brains included in the DS evaluation, whereas the rest were followed by an overnight immersion in $10 \%$ buffered formalin.

\section{Magnetic resonance evaluation}

After the functional evaluation, brains fixed by the standard protocol were randomly selected to perform MRI, obtaining 8 animals for each group. MRI was performed on fixed brains using a 7T animal MRI scanner (BrukerBioSpin MRI GmbH, Ettlingen, Germany). High-resolution three-dimensional T2-weighted and diffusion-weighted images (DWI) were acquired. Diffusion Tensor model was then fitted and fractional anisotropy (FA) was estimated in each voxel. Automatic parcellation of the subjects' brain was performed based on the New Zealand Rabbit MRI Atlas [31]. Brain FA-weighted network for each subject was extracted and infrastructure (average strength), integration (weighted global efficiency) and segregation (weighted local efficiency) were assessed. In addition, median FA from the hippocampal regions was computed for each hemisphere, as well as median FA of the reconstructed streamlines crossing hippocampal regions.

\section{Histology assessment}

a) Dendritic spine evaluation: 15 to 20 basal dendrites from each subject's hemisphere were selected to be evaluated from CA1 of the dorsal hippocampus (see Figure 1) using the Helios Gene Gun System (Bio-Rad) [32]. CA1 was selected for this analysis, as it has been described to be the hippocampal area that receives the major input connections [33,34]. The 
Density of DS (number of spines/ $\mu \mathrm{m}$ ) was calculated, including a final sample of 138 dendrites from controls, 155 dendrites from IUGR and 128 t-IUGR.

b) Perineuronal nets (PNNs): 16 brains fixed by the standard protocol explained previously (controls: $n=4$; IUGR: $n=6$; t-IUGR: $n=6$ ) were randomly selected to evaluate the PNNs. This analysis was done using lectin histochemistry Wisteria Floribunda (WFA) -binding and quantifying the average density of immunolabeling (contact $/ \mu \mathrm{m}^{2}$ ) from CA3 of the hippocampus (see Figure 1). Similarly to previous works [35], CA3 area from the hippocampus was preferred to analyze PNNs since the greatest amount of WFA staining was observed in comparison to the CA1 area.

For a more detailed description of MRI processing and histology assessment, see Supplementary Material.

\section{Statistics}

For quantitative variables, normality was assessed by Shapiro-Wilk Test and homoscedasticity by Levene's Test. Results were expressed as mean and standard deviation (SD) for normal variables; whereas median and interquartile rates (IQR) were used in non-normal variables. In neonatal data, normaldistributed quantitative variables were analyzed by $\mathrm{t}$-test or with Kruskal-Wallis test when needed. For categorical variables, Chi-squared test was used. At the long-term period, differences between cases and controls were analyzed using general lineal model (GLM) adjusting by gender. In this case, when the null hypothesis in Shapiro-Wilk or in Levene's Test was rejected, log-transformation was performed prior to the analysis. Finally, association of network features, DS and PNNs with functional results was performed by means of partial correlation 
analysis. The software package STATA13.0 was used for the statistical analyses. Significance was declared at $p<0.05$.

\section{RESULTS}

\section{Survival and growth parameters}

Stillbirth was higher in non-treated IUGR compared with controls (55\% vs. $8 \%, p<0.001$ ), with no statistical differences when comparing t-IUGR and IUGR ( $45 \%$ vs. $55 \%, p=0.125$ ). Birth weight was significantly lower in IUGR than in controls (33.6g (SD 1.3) vs. 46.7g (SD 1.3), $\mathrm{p}<0.001)$, without any significant difference between IUGR groups (IUGR 34.3g (SD 2.4)) and t-IUGR (33.6g (SD 1.3), $p=0.871)$. At $+60 \mathrm{P}$, no differences between groups were observed either in weight or in gender distribution.

\section{Functional results}

At the neonatal period, non-treated IUGR pups showed poorer results in almost all the neurodevelopmental parameters assessed when compared to controls, whereas no significant differences were observed when comparing IUGR and t-IUGR groups (Table S1).

At the long-term period, non-treated IUGR animals presented functional impairments compared to controls, showing a trend to present reduced learning skills although not being statistically significant, significant memory impairment and a higher degree of anxiety (lower DI and less time exploring the internal area). Of note, t-IUGR animals presented an improvement in memory and anxiety trait when compared with IUGR subjects (Figure 2). 


\section{MRI results}

Analysis of global network features evidenced a significant decrease in average strength, global and local efficiencies in non-treated IUGR when compared to controls. A significant increase with respect to IUGR was observed in all these variables in the IUGR group in which therapy was applied (Figure 3). Regional analysis revealed no significant differences in the brain volume of the hippocampus within the different groups (Table S2). Analysis of regional FA parameters showed reduced median FA in both the left hippocampus region and the fibers crossing it in non-treated IUGR animals with respect to controls. Interestingly, when compared with IUGR group, t-IUGR animals showed a significant increase in these parameters with similar values to the control group (Figure 4). In addition, significant correlations were observed between global network features and neurobehavioral results, especially in the OFBT and ORT variables (Supplementary Table S3).

\section{Histology results: DS and PNNs}

Non-treated IUGR animals presented a significant decrease in DS density when compared to controls, with a significant increase in the t-IUGR animals compared to IUGR (Figure 5a). Similarly, non-treated IUGR animals presented a significant decrease in PNNs immunoreactivity when compared to controls, with trends to increase and normalize to control levels if the therapy has been applied (Figure 5b). Finally, no significant correlations were observed between DS or PNNs and neurologic performance (Supplementary Table S3). 


\section{DISCUSSION}

To our knowledge, this is the first study using connectivity analysis at the whole brain and cellular level to show an altered brain connectivity following IUGR that persists beyond adolescence. We hypothesize that these structural brain changes could underpin the neurobehavioral disabilities observed in our animal model. Additionally, we demonstrated that exposure to an enriched environment during early postnatal period ameliorates these effects on brain development after IUGR, partially recovering connectivity and neurobehavioral impairments.

In this study, advanced ex vivo MRI combined with histological markers of neuronal connectivity described changes in brain connectivity that persist up to the long-term period after IUGR. MRI results support previous findings in the rabbit model showing impaired global network infrastructure, integration, and segregation evidenced by a reduction in FA-weighted strength, global and local efficiencies [13,28]. Likewise, alterations in brain networks have been previously described in humans to persist in childhood and early adolescence $[11,12,14,36]$. Apart from global changes, regional analysis of hippocampus was also explored due to its important role in memory and cognition in animals and humans [37] and for their vulnerability to IUGR [38]. Regional analysis showed decreased FA in the left hippocampus together with a reduction of median FA of fibers passing through the hippocampus. These results suggested the presence of less mature connections since FA has been related to axonal packing, neuronal density, and myelination of fiber tracts [39]. Predominant changes affecting one of the brain hemispheres is coherent with the idea that 
some neural functions tend to be more dominant in one hemisphere than in the other [40]. In particular, left hippocampus has been described to be related with memory and neurobehavioral impairments in the considered rabbit model [29] as well as in rodents $[41,42]$.

Regarding histological assessment, a significant reduction of DS and PNNs density in CA1 and CA3 hippocampal pyramidal neurons was observed. Both DS and PNNs have been involved in the regulation of synaptic connectivity and plasticity [43-46]. Our results showing decreased levels of DS in IUGR rabbits are in line with previously described studies on guinea pig and sheep model showing changes in DS density and morphology along with changes in synaptic receptors after acute and chronic intrauterine insults [16, 47-49]. On the contrary, although there is growing interest in the description of PNNs alterations related with specific brain diseases such as Alzheimer, schizophrenia and epilepsy [45, 50], the pattern of alterations in the PNNs related with IUGR had not been previously evaluated. It has been described that normal completion of PNNs guarantees, in the adult brain, the stability of the established neuronal connections [51]. Therefore, decreased PNNs density in the IUGR animals in CA3 suggests less consolidated connections in the hippocampus, which is coherent with the lower amount of DS found in CA1. Indeed, preliminary evidence suggests that reduction of synapses expressed as reduced DS is associated with reduced PNNs formation [52]. These changes at the cellular level were related to MRI findings, especially with regional reduction of FA in the hippocampus and white matter tracts connection. 
Our results demonstrate for the first time that an early postnatal strategy based on EE can improve behavioral performance and brain connectivity after IUGR. This is in agreement with previous basic research where the potential of EE as a non-invasive rehabilitation strategy has been established in rat models of hypoxic-ischemic neonatal injury [53] and prenatal exposition to alcohol [54]. Previous evidence have demonstrated the beneficial effects of EE in animal models as a modulator of key sites of brain connectivity $[18,22]$. Moreover, our data go in line with clinical evidence showing that NIDCAP program (physical and emotional support to premature infant during neonatal intensive care unit admission) is related with neurobehavioral and structural improvement in severe IUGR preterm infants [55]. Together with positive effects on function, we also observed a recovery in brain connectivity with improved global network feature and increased DS density and PNNs. These changes at the cellular level after EE have also been shown in a rat model of neonatal hypoxia-ischemia with preserved DS [53] and in addiction based model with increased PNNs density [56]. The improvement at both behavioral and structural level is crucial to demonstrate the actual effect of EE therapy identifying those functions and regions more sensitive to its effects and to support its implementation in clinical conditions.

This study has some strengths and limitations that merit comment. Despite the limitations of animal research, one of its major strengths is the potential to test therapies and the transferability of these results to humans. On one hand, rabbit brain shows a timing of perinatal brain white matter maturation closer to humans compared to other species [26]. Regarding the IUGR model, either 
perinatal results and the reported neonatal and long-term neurodevelopmental impairments are in good agreement with the literature for this model $[13,24,28,29]$ and also with clinical observations [2-6,8]. Regarding histological assessment, DS are plastic structures and they are constantly subjected to external inputs [57]. However, the experimental setting reduces this variability. Indeed, other features of structural synaptic plasticity, such as dendritic spine morphology and distribution patterns, dendritic branching and length, or analyses of the specific perineuronal net component of the extracellular may be of equal interest to be evaluated in the IUGR and may give additional insights in our results. Further studies should be considered to evaluate all these additional features of structural synapsis and also evaluate them in other brain areas different from the hippocampus, as imaging studies of IUGR have revealed reduced volumes and diffusion $\mathrm{MRI}$ changes of other grey matter structures [16]. Finally, due to sample size, we acknowledge that we were underpowered for some of the comparisons. In order to quantify the power of the reported variables, a supplementary table reporting the mean and risk differences, as appropriate, and its 95\% confidence interval was provided (Table S4).

Hence, by combining MRI with histological results we observed that IUGR may disrupt the normal pattern of brain development affecting special key sites for synaptic activity. These connectivity impairments either at global or at the cellular level that persist up to the long-term period may explain, at least in part, the basis for the neurodevelopmental disorders associated with IUGR. Environmental enrichment during the early postnatal period could ameliorate the effect of prenatal insults on neurodevelopment, with functional and structural 
changes that partially recovers normal conditions. Overall, our results reinforce the notion that environmental factors during critical periods of neurodevelopment could modify development and predispose the individual to

lifelong health problems or enhance it. Further evaluation of EE effects in a clinical setting is needed to explore its real effects and also to determine the exact moment to apply such strategy in IUGR infants.

\section{Acknowledgments}

We would like to acknowledge Guadalupe Soria, Xavi Lopez and Raúl Tudela from Experimental $7 \mathrm{~T} \mathrm{MRI}$ Unit of the Institut d'Investigacions Biomèdiques August Pi I Sunyer (IDIBAPS) for their help in the performance of MRI acquisitions. We are also indebted to Isabel Salas and the rest of the animal housing staff from Hospital Sant Joan de Déu - Animal Housing for their sensitivity in the care of the animal during the procedures. The Image Registration Toolkit was used under Licence from Ixico Ltd. 


\section{References}

[1] Bustos JC, Gonzalez V, Sepulveda W: Umbilical Artery Half-Peak Systolic Velocity Deceleration Time in Fetal Growth Restriction. Fetal Diagn Ther $2016 ; 40: 128-134$.

[2] Geva R, Eshel R, Leitner Y, Fattal-Valevski A, Harel S: Memory functions of children born with asymmetric intrauterine growth restriction. Brain Res 2006;1117(1):186-94.

[3] Geva R, Eshel R, Leitner Y, Valevski AF, Harel S: Neuropsychological outcome of children with intrauterine growth restriction: a 9-year prospective study. Pediatrics 2006;118(1):91-100.

[4] Alati R, Najman JM, O'Callaghan M, Bor W, Williams GM, Clavarino A: Fetal growth and behaviour problems in early adolescence: Findings from the Mater University Study of Pregnancy. Int J Epidemiol 2009;38(5):1390-400.

[5] Larroque B, Bertrais S, Czernichow P, Léger J: School difficulties in 20year-olds who were born small for gestational age at term in a regional cohort study. Pediatrics 2001;108(1):111-5.

[6] Tideman E, Marsál K, Ley D: Cognitive function in young adults following intrauterine growth restriction with abnormal fetal aortic blood flow. Ultrasound Obstet Gynecol 2007;29(6):614-8.

[7] Figueras F, Oros D, Cruz-Martinez R, Padilla N, Hernandez-Andrade E, Botet F, Costas-Moragas C, Gratacos E: Neurobehavior in term, small-forgestational age infants with normal placental function. Pediatrics 2009;124(5):e934-41.

[8] Kady S, Gardosi J: Perinatal mortality and fetal growth restriction. Best Pract Res Clin Obstet Gynaecol 2004;18(3):397-410. 
[9] MacKay DF, Smith GCS, Dobbie R, Cooper SA, Pell JP: Obstetric factors and different causes of special educational need: Retrospective cohort study of 407503 schoolchildren. BJOG An Int J Obstet Gynaecol 2013;120(3):297-307 .

[10] Ment LR, Hirtz D, Hüppi PS: Imaging biomarkers of outcome in the developing preterm brain. Lancet Neurol 2009;8(11):1042-55.

[11] Fischi-Gómez E, Muñoz-Moreno E, Vasung L, Griffa A, Borradori-Tolsa C, Monnier M, Lazeyras F, Thiran JP, Hüppi PS: Brain network characterization of high-risk preterm-born school-age children. Neurolmage: Clinical 2016;11:195-209.

[12] Fischi-Gómez E, Vasung L, Meskaldji D-E, Lazeyras F, Borradori-Tolsa C, Hagmann P, Barisnikov K, Thiran JP, Hüppi PS: Structural brain connectivity in school-age preterm infants provides evidence for impaired networks relevant for higher order cognitive skills and social cognition. Cereb Cortex 2015 Sep;25(9):2793-805.

[13] Batalle D, Muñoz-Moreno E, Arbat-Plana A, Illa M, Figueras F, Eixarch E, Gratacós E: Long-term reorganization of structural brain networks in a rabbit model of intrauterine growth restriction. Neuroimage 2014;100:24-38.

[14] Muñoz-Moreno E, Fischi-Gomez E, Batalle D, Borradori-Tolsa C, Eixarch E, Thiran J-P, Gratacós E, Hüppi PS: Structural brain network reorganization and social cognition related to adverse perinatal condition from infancy to early adolescence. Frontiers in Neuroscience 2016;10:560.

[15] Van Den Heuvel MP, Scholtens LH, De Reus MA, Kahn RS: Associated microscale spine density and macroscale connectivity disruptions in schizophrenia. Biol Psychiatry 2016;80(4):293-301. 
[16] Dean JM, McClendon E, Hansen K, Azimi-Zonooz A, Chen K, Riddle A, Gong X, Sharifnia E, Hagen M, Ahmad T, Leigland LA, Hohimer AR, Kroenke CD, Back SA: Prenatal cerebral ischemia disrupts MRI-defined cortical microstructure through disturbances in neuronal arborization. Sci Transl Med 2013;5(168):168ra7.

[17] Rao MR, Hediger ML, Levine RJ, Naficy AB, Vik T: Effect of breastfeeding on cognitive development of infants born small for gestational age. Acta Paediatr 2002; 91: 267- 74.

[18] Rampon C, Tsien JZ: Genetic analysis of learning behavior-induced structural plasticity. Hippocampus 2000;10(5):605-9.

[19] Chapillon P, Patin V, Roy V, Vincent A, Caston J: Effects of pre- and postnatal stimulation on developmental, emotional, and cognitive aspects in rodents: A review. Dev Psychobiol 2002;41(4):373-87.

[20] Fares RP, Belmeguenai A, Sanchez PE, Kouchi HY, Bonnet C, Bodennec J, Morales A, Georges B, Bonnet C, Bouvard S, Sloviter RS, Bezin L: Standardized Environmental Enrichment Supports Enhanced Brain Plasticity in Healthy Rats and Prevents Cognitive Impairment in Epileptic Rats 2013;8(1):e53888.

[21] Rosenzweig MR: Aspects of the search for neural mechanisms of memory. Annu Rev Psychol 1996;47:1-32.

[22] Rampon C, Tang YP, Goodhouse J, Shimizu E, Kyin M, Tsien JZ: Enrichment induces structural changes and recovery from nonspatial memory deficits in CA1 NMDAR1-knockout mice. Nat Neurosci 2000;3(3):238-44.

[23] Johnston $M$ V: Plasticity in the developing brain: implications for rehabilitation. Dev Disabil Res Rev 2009;15(2):94-101. 
[24] Eixarch E, Figueras F, Hernández-Andrade E, Crispi F, Nadal A, Torre I, Oliveira S, Gratacós E: An experimental model of fetal growth restriction based on selective ligature of uteroplacental vessels in the pregnant rabbit. Fetal Diagn Ther 2009;26(4):203-11.

[25] Baumans V, Van Loo PLP: How to improve housing conditions of laboratory animals: The possibilities of environmental refinement. Vet $\mathrm{J}$ 2013;195(1):24-32.

[26] Derrick M, Luo NL, Bregman JC, Jilling T, Ji X, Fisher K, Gladson CL, Beardsley DJ, Murdoch G, Back SA, Tan S: Preterm fetal hypoxia-ischemia causes hypertonia and motor deficits in the neonatal rabbit: a model for human cerebral palsy? J Neurosci 2004;24(1):24-34.

[27] Tan S, Drobyshevsky A, Jilling T, Ullman LM, Englof I, Derrick M: Model of Cerebral Palsy in the Perinatal Rabbit. J Child Neurol 2005;20(12):972-9.

[28] Illa M, Eixarch E, Muñoz-Moreno E, Batalle D, Leal-Campanario R, Gruart A, Delgado-García JM, Figueras F, Gratacós E: Neurodevelopmental Effects of Undernutrition and Placental Underperfusion in Fetal Growth Restriction Rabbit Models. Fetal Diagn Ther 2017 in press.

[29] Illa M, Eixarch E, Batalle D, Plana-Arbat A, Muñoz-Moreno E, Figueras F, Gratacós E: Long-term functional outcomes and correlation with regional brain connectivity by MRI diffusion tractography metrics in a near-term rabbit model of intrauterine growth restriction. PLoS One 2013 15;8(10):e76453.

[30] de Bruin N, Pouzet B: Beneficial effects of galantamine on performance in the object recognition task in Swiss mice: deficits induced by scopolamine and by prolonging the retention interval. Pharmacol Biochem Behav 2006;85(1):253-60. 
[31] Muñoz-Moreno E, Arbat-Plana A, Batalle D, Soria G, Illa M, PratsGalino A, Eixarch E, Gratacos E: A Magnetic Resonance Image Based Atlas of the Rabbit Brain for Automatic Parcellation. PLoS One 2013 Jul 2;8(7):e67418.

[32] Grutzendler J, Tsai J, Gan WB: Rapid labeling of neuronal populations by ballistic delivery of fluorescent dyes. Methods 2003;30(1):79-85.

[33] Takács VT, Klausberger T, Somogyi P, Freund TF, Gulyás Al: Extrinsic and local glutamatergic inputs of the rat hippocampal CA1 area differentially innervate pyramidal cells and interneurons. Hippocampus 2012;22(6):1379-91.

[34] Spruston N: Pyramidal neurons: dendritic structure and synaptic integration. Nat Rev Neurosci 2008;9(3):206-21.

[35] Hylin MJ, Orsi SA, Moore AN, Dash PK: Disruption of the perineuronal net in the hippocampus or medial prefrontal cortex impairs fear conditioning. Learn Mem 2013;20(5):267-73.

[36] Batalle D, Eixarch E, Figueras F, Muñoz-Moreno E, Bargallo N, Illa M, Acosta-Rojas R, Amat-Roldan I, Gratacos E: Altered small-world topology of structural brain networks in infants with intrauterine growth restriction and its association with later neurodevelopmental outcome. Neuroimage 2012;60(2):1352-66.

[37] Eichenbaum H: Hippocampus: Cognitive Processes and Neural Representations that Underlie Declarative Memory. Neuron 2004;44(1):109-20. [38] Mallard C, Loeliger M, Copolov D, Rees S: Reduced number of neurons in the hippocampus and the cerebellum in the postnatal guinea-pig following intrauterine growth-restriction. Neuroscience 2000;100(2):327-33.

[39] Sen PN, Basser PJ: A model for diffusion in white matter in the brain. Biophys J 2005;89(5):2927-38. 
[40] Duboc V, Dufourcq P, Blader P, Roussigné M: Asymmetry of the brain: development and implications. Annu Rev Genet 2015;49(1):647-72.

[41] Shipton OA, El-Gaby M, Apergis-Schoute J, Deisseroth K, Bannerman DM, Paulsen O, Kohl MM: Left-right dissociation of hippocampal memory processes in mice. Proc Natl Acad Sci 2014;111(42):15238-43.

[42] $\mathrm{Hu} H$, Su L, Xu YQ, Zhang H, Wang LW: Behavioral and [F-18] fluorodeoxyglucose micro positron emission tomography imaging study in a rat chronic mild stress model of depression. Neuroscience 2010;169(1):171-81.

[43] Yuste R: Dendritic spines and distributed circuits. Neuron $2011 ; 71(5): 772-81$.

[44] Harris KM, Kater SB: Dendritic spines: cellular specializations imparting both stability and flexibility to synaptic function. Annu Rev Neurosci $1994 ; 17(1): 341-71$.

[45] Dzyubenko E, Gottschling C, Faissner A: Neuron-glia interactions in neural plasticity: contributions of neural extracellular matrix and perineuronal nets. Neural Plast 2016;2016:5214961.

[46] Geissler M, Gottschling C, Aguado A, Rauch U, Wetzel CH, Hatt H, Faissner A: Primary hippocampal neurons, which lack four crucial extracellular matrix molecules, display abnormalities of synaptic structure and function and severe deficits in perineuronal net formation. J Neurosci 2013;33(18):7742-55.

[47] Dieni S, Rees S: Dendritic morphology is altered in hippocampal neurons following prenatal compromise. J Neurobiol 2003;55(1):41-52.

[48] McClendon E, Chen K, Gong X, Sharifnia E, Hagen M, Cai V, Shaver DC, Riddle A, Dean JM, Gunn AJ, Mohr C, Kaplan JS, Rossi DJ, Kroenke CD, 
Hohimer AR, Back SA: Prenatal cerebral ischemia triggers dysmaturation of caudate projection neurons. Ann Neurol 2014;75(4):508-24.

[49] Piorkowska K, Thompson J, Nygard K, Matushewski B, Hammond R, Richardson B: Synaptic development and neuronal myelination are altered with growth restriction in fetal guinea pigs. Dev Neurosci 2014;36(6):465-76.

[50] Cabungcal JH, Steullet P, Kraftsik R, Cuenod M, Do KQ: Early-life insults impair parvalbumin interneurons via oxidative stress: Reversal by $\mathrm{N}$ acetylcysteine. Biol Psychiatry 2013;73(6):574-82.

[51] Wang D, Fawcett $\mathrm{J}$ : The perineuronal net and the control of cns plasticity. Cell Tissue Res 2012;349(1):147-60.

[52] Faissner A, Pyka M, Geissler M, Sobik T, Frischknecht R, Gundelfinger ED, Seidenbecher C: Contributions of astrocytes to synapse formation and maturation - Potential functions of the perisynaptic extracellular matrix. Brain Res Rev 2010;63(1-2):26-38.

[53] Jiménez J, Ferrary B, Maidana P, Diaz R, Hermel E, Achaval M, Netto CA, Pereira LO: Effects of daily environmental enrichment on behavior and dendritic spine density in hippocampus following neonatal hypoxia - ischemia in the rat. Exp Neurol 2013;241:25-33.

[54] Hannigan JH, Berman RF: Amelioration of fetal alcohol-related neurodevelopmental disorders in rats: Exploring pharmacological and environmental treatments. Neurotoxicol Teratol 2000;22(1):103-11.

[55] Als H, Duffy FH, McAnulty G, Butler SC, Lightbody L, Kosta S, Weisenfeld NI, Robertson R, Parad RB, Ringer SA, Blickman JG, Zurakowski D, Warfield SK: NIDCAP improves brain function and structure in preterm 
infants with severe intrauterine growth restriction. J Perinatol 2012;32(10):797803.

[56] Slaker M, Barnes J, Sorg BA, Grimm JW: Impact of environmental enrichment on perineuronal nets in the prefrontal cortex following early and late abstinence from sucrose self-administration in rats. PLoS One 2016;11(12):e0168256.

[57] Engert F, Bonhoeffer T: Dendritic spine changes associated with hippocampal long-term synaptic plasticity. Nature 1999;399:66-70. 


\section{Figures legends}

Figure 1: Graphical representation of the study design and methods

A) Illustrative images and scheme of IUGR induction. After the breastfeeding period ( $>30$ postnatal days), a cohort of IUGR animals was randomized into the environmental enrichment protocol (t-IUGR) whereas the rest were housed conventionally.

B) Illustrative images of neurobehavioral tests applied. At postnatal day +1 , tone, spontaneous locomotion, reflex motor activity, coordination of suck and swallow and motor responses to olfactory stimuli were assessed. At postnatal day +60 and $+70 \mathrm{P}$, Skinner test, OFBT, and ORT were applied.

C) Magnetic resonance imaging. Fixed brains were scanned, obtaining anatomical and diffusion-weighted images. FA brain networks were extracted and global graph theory features were applied. Regional analysis was done with mean FA of the hippocampus and mean FA of fibers crossing it.

D) Histology. Connectivity characteristics of hippocampal regions were assessed including dendritic spine density and perineuronal nets evaluation.

Abbreviations: Cont: control; IUGR: intrauterine growth restriction; t-IUGR: Treated intrauterine growth restriction animals; OFBT: open field behavioral test; ORT: object recognition task.

\section{Figure 2: Functional evaluation at long-term period}

A) Percentage of learning in the study groups (controls, IUGR, and t-IUGR) obtained from the Skinner test.

B) Discriminatory index in the study groups (controls, IUGR, and t-IUGR) from the ORT. 
C) Time spent in the internal area in the study groups (controls, IUGR, and tIUGR) from the OFBT.

Abbreviations: IUGR= intrauterine growth restriction; $t-I U G R=$ Treated

intrauterine growth restriction animals; OFBT: open field behavioral test; ORT:

object recognition task. ${ }^{*} p<0.05$ statistical significance.

\section{Figure 3: Global FA network features}

Global fractional anisotropy (FA) network features in the study groups (controls, IUGR and t-IUGR). Networks features included average strength, global and local efficiency of weighted FA network.

Abbreviations: IUGR= intrauterine growth restriction; $t-I U G R=$ Treated intrauterine growth restriction animals. ${ }^{*} p<0.05$ statistical significance.

\section{Figure 4: MRI regional Hippocampus}

Median FA from the hippocampal regions and from the reconstructed fibers crossing hippocampal regions was evaluated in the study groups (controls, IUGR, and t-IUGR).

Abbreviations: IUGR= intrauterine growth restriction; $\mathrm{t}$-IUGR=Treated intrauterine growth restriction animals. ${ }^{*} p<0.05$ statistical significance.

\section{Figure 5: Histology}

A) Dendritic spine analysis:

A.1 Density of dendritic spine from basal dendrites of CA1 pyramidal neurons from the dorsal hippocampus in the study groups (controls, IUGR, and t-IUGR). A.2 Illustrative images of the dendritic spine density in the study groups 
(controls, IUGR, and t-IUGR).

B) Perineuronal nets analysis:

A.1 Average density of immunolabelling from hippocampus CA3 zone in the study groups (controls, IUGR, and t-IUGR).

A.2 Illustrative images of the immunolabelling of perineuronal nets in the study groups (controls, IUGR, and t-IUGR).

Abbreviations: IUGR= intrauterine growth restriction; $\mathrm{t}-\mathrm{IUGR}=$ Treated intrauterine growth restriction animals. ${ }^{*} p<0.05$ statistical significance.

\section{Supplementary material}

Supplementary Materials and Methods. Brain network analysis and Histology assessment methodology.

Supplementary Table S1: Functional results at the neonatal period in study groups.

Supplementary Table S2: Hippocampus volume results.

Supplementary Table S3: Mean correlation coefficients between neurobehavioral items results versus FA-weighted network features, DS and PNNs.

Supplementary Table S4: Mean or risk difference, as appropriate, and its 95\% confidence interval $(\mathrm{Cl})$ for reported variables. 


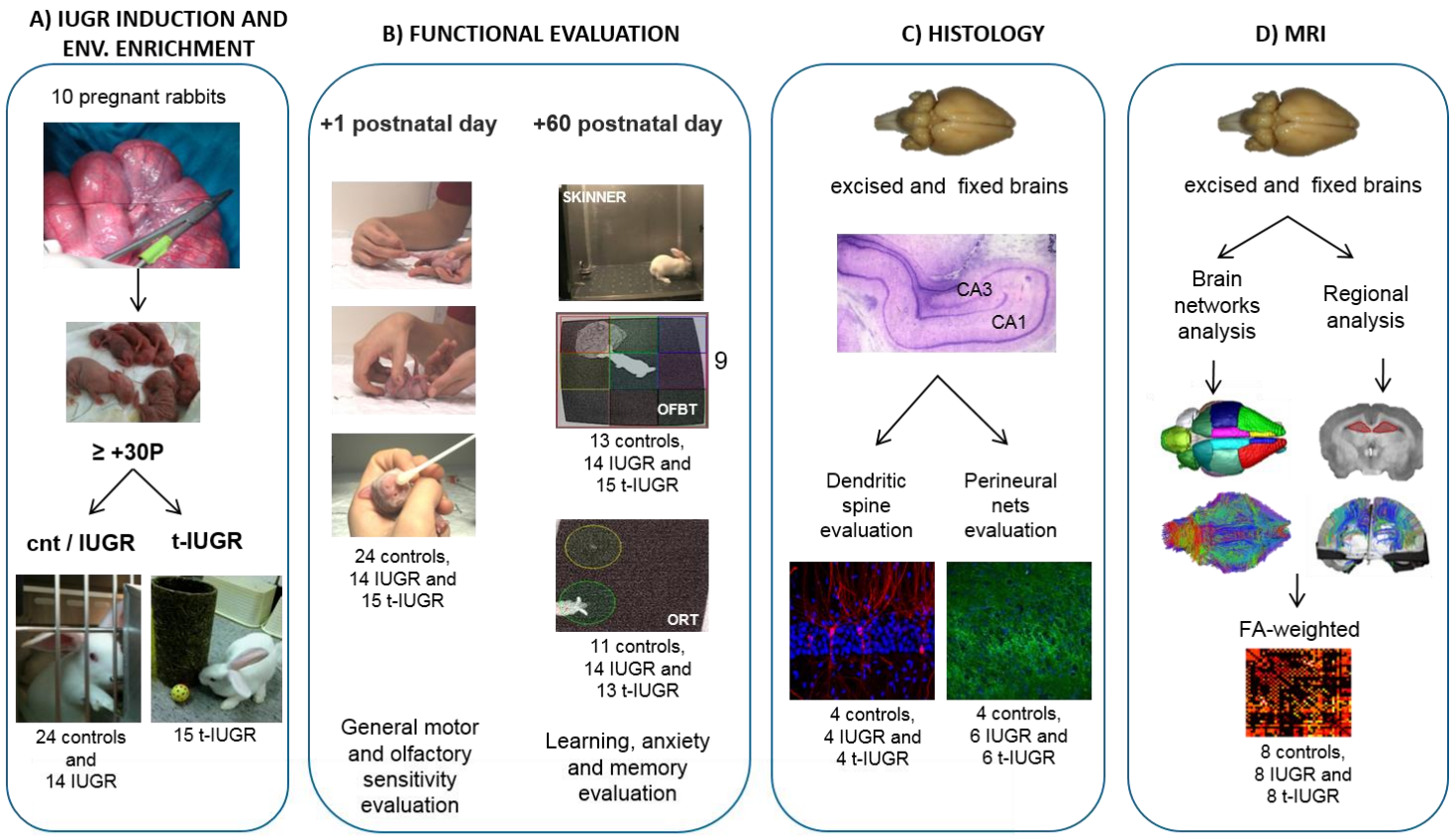

Figure 1.

a) Skinner test

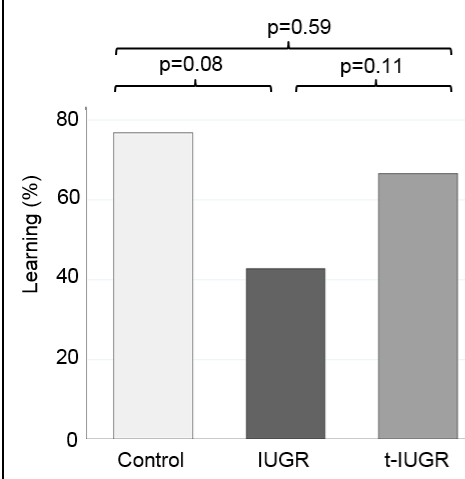

b) ORT

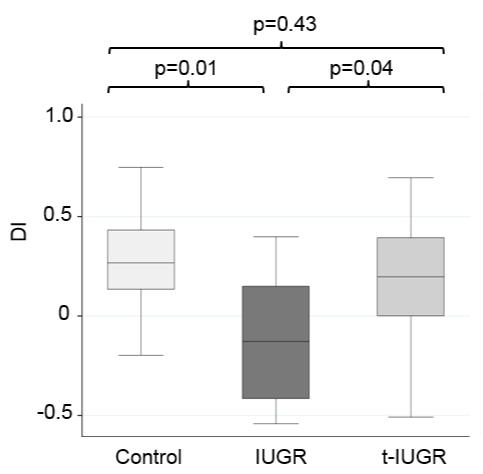

c) OFBT

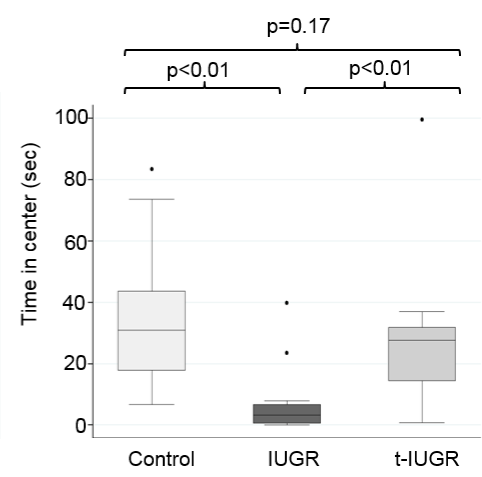

Figure 2. 


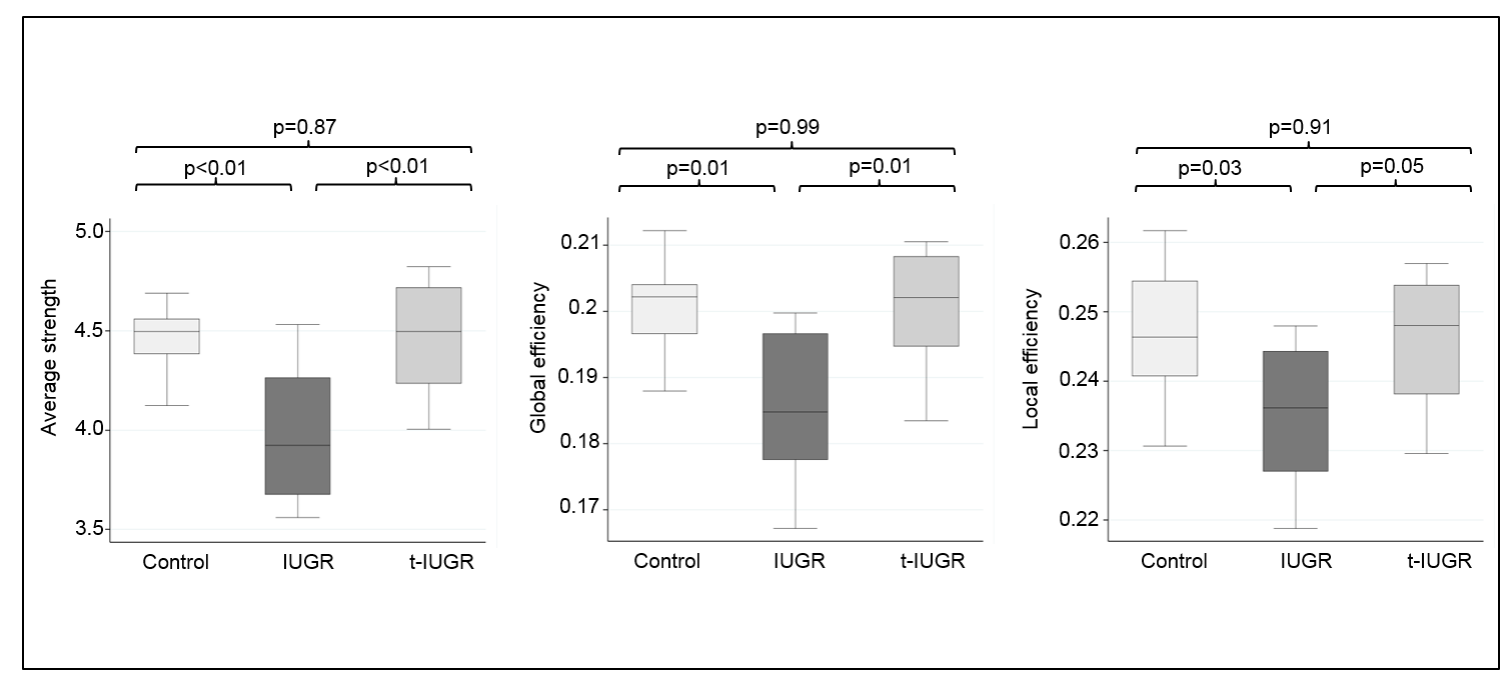

Figure 3.

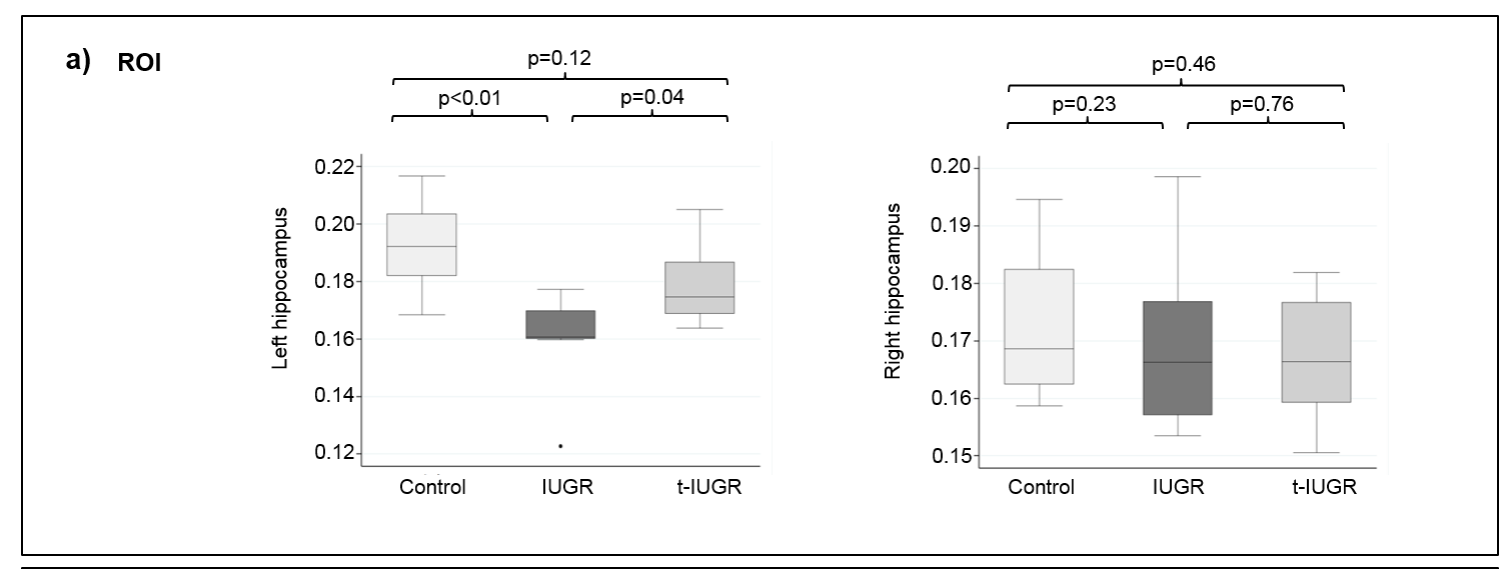

b) Fibers
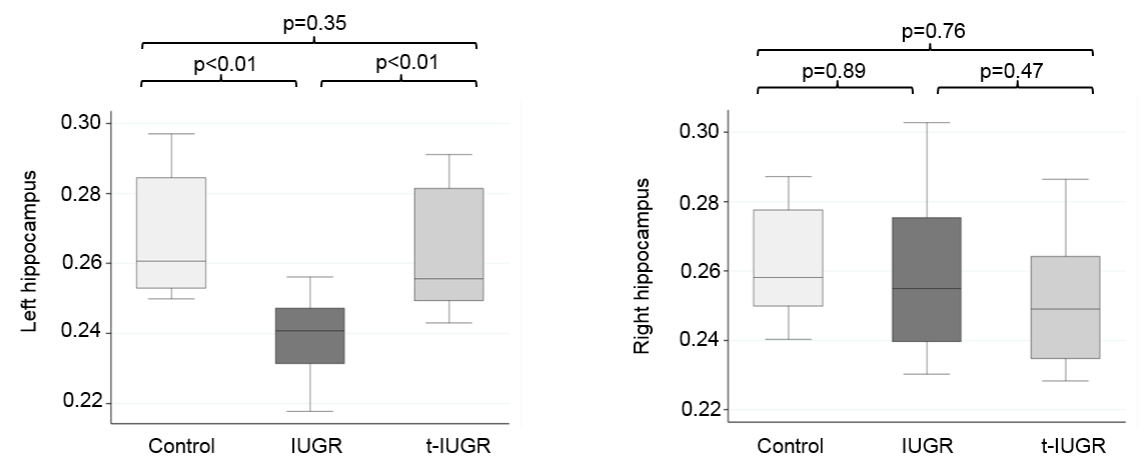

Figure 4. 


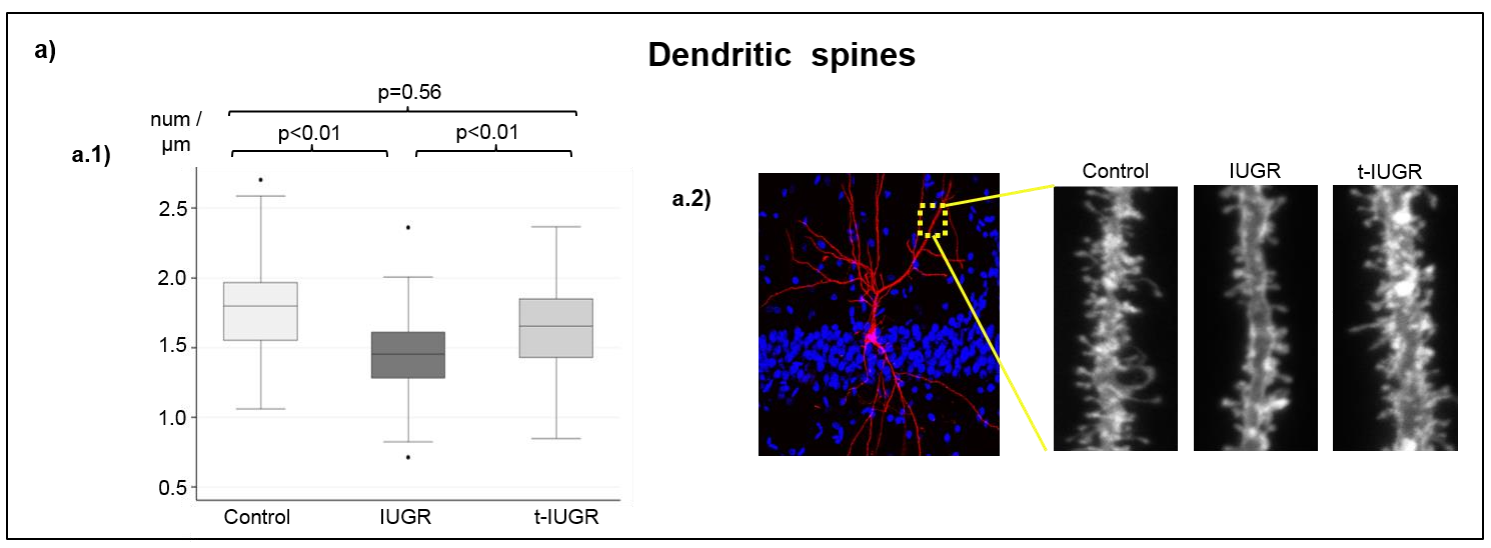

b)

Perineural nets

b.1) $\quad \begin{gathered}\text { contact } / \\ { } }\end{gathered}$

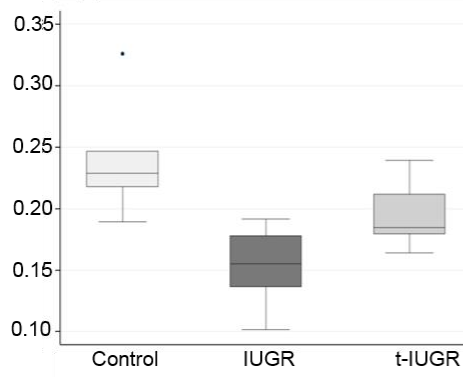

b.2)
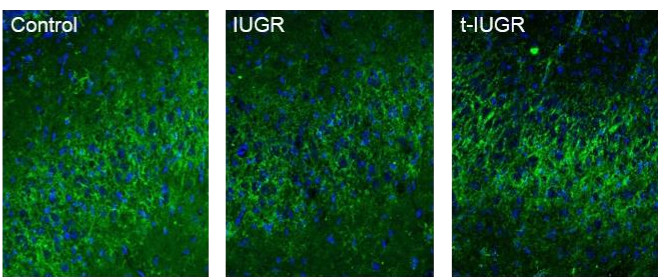

Figure 5. 